\title{
Contexto Emocional Negativo e Processamento Consciente na Produção de Falsas Memórias em Tarefas de Reconhecimento
}

\author{
Negative Emotional Context and Conscious Processing in the Production \\ of False Memories in Recognition Tasks
}

\author{
Tin Po Huang* \& Gerson Américo Janczura \\ Universidade de Brasilia, Brasília, Distrito Federal, Brasil
}

\begin{abstract}
Resumo
Este trabalho investigou a produção de falsas memórias em relação a três fatores: tipos de processamento, carga afetiva das listas DRM e contexto emocional. Foram testadas quatro hipóteses experimentais: (a) índices superiores de falsas memórias ocorrerão em tarefas que demandam maior participação de processamento consciente, (b) aumento de falsas memórias em contexto emocional, (c) maior falsificação de memória nas listas DRM com cargas negativas e (d) mais falsas recordações na condição processamento consciente, listas negativas e contexto de valência desagradável. Participaram 150 estudantes e o contexto emocional foi manipulado pela inserção de fotografias do IAPS no paradigma DRM. Os resultados confirmaram as hipóteses do estudo e são discutidos segundo teorias de redes e da teoria do traço difuso.
\end{abstract}

Palavras-chave: Falsas memórias, processamento consciente e automático, DRM, contexto emocional.

\begin{abstract}
The present study investigated the production of false memories in relation to three independent factors: processing type, emotional context and affective valence of Deese-Roediger-McDermott (DRM) lists. Four experimental hypotheses were tested: (a) higher rates of false memories will occur in tasks that require greater participation of conscious processing; (b) increase of false memories in emotional contexts; (c) greater falsification of memory in DRM lists with negative valence; and (d) more false memories in conscious processing, negative lists and unpleasant valence contexts. One hundred and fifty university students participated in the experiment. The emotional context was manipulated by inserting pictures from the International Affective Picture System (IAPS) within DRM lists. Results confirmed the experimental hypotheses and were discussed considering theories of nets and conjoint activations.

Keywords: False memories, conscious and automatic processing, DRM, emotional context.
\end{abstract}

O fenômeno de falsas lembranças ou ilusões de memória se refere às lembranças de eventos ou informações que na realidade não ocorreram ou à recordação de fatos que ocorreram de uma forma bastante diferente de como realmente aconteceram (Roediger \& McDermott, 1995). Numa tentativa de entender o fenômeno, pesquisadores estão investigando vários fatores que podem inibir ou aumentar a produção de falsas memórias (Gallo, 2006). Algumas questões estudadas focalizam o papel do processamento automático ou consciente na produção do fenômeno (Cotel, Gallo, \& Seamon, 2008) ou, ainda, se a carga emocional das experiências contribui na formação de

\footnotetext{
*Endereço para correspondência: Departamento de Processos Psicológicos Básicos, Instituto de Psicologia, Universidade de Brasília, PPB Asa Norte, Brasília, DF, Brasil 70910-900. E-mail: tinhuang9@gmail.com e janczura@unb.br
}

falsas memórias (Brainerd, Stein, Oliveira, Rosenkohl, \& Reyna, 2008). A influência da emoção nas falsas memórias é especialmente desafiadora teórica e metodologicamente e, recentemente, tem sido um dos focos da pesquisa. Quais dimensões emocionais e em que tipos de situações o estado emocional de um indivíduo afetará a acurácia de suas lembranças? Além disso, qual é a contribuição de contextos emocionais na memorização da informação? É razoável supor que palavras associadas semanticamente sejam influenciadas por contextos emocionais, produzindo ilusões de memória?

Esta pesquisa contribui na compreensão dessas questões investigando experimentalmente a influência da valência dos estímulos verbais, do tempo de apresentação (processamento automático e consciente) e do contexto emocional na produção de falsas memórias.

A emoção é conceituada como possuindo um aspecto de reatividade, geralmente breve, intensa e circunscrita, 
relacionada a um evento ambiental específico. Ela tem sido utilizada para se referir a um estado afetivo presente na fase de codificação ou evocação da memória. Sob o ponto de vista experimental, a emoção refere-se ao estado afetivo/fisiológico que um indivíduo apresenta durante um teste de memória. A emoção humana inclui, pelo menos, duas dimensões independentes: o alerta e a valência. A dimensão alerta se refere à intensidade ou grau que varia da calma, relaxamento até a excitação. Já a dimensão valência reflete uma variação do agradável ao desagradável, ou seja, a qualificação da emoção (Ellis \& Moore, 1999). Sabe-se que a valência da emoção pode distorcer a lembrança da memória humana, na fase de codificação, em duas situações principais: informações emocionais são mais falsamente reconhecidas que informações não emocionais (Doerksen \& Shimamura, 2001; Maratos, Allan, \& Rugg, 2000) e informações não-emocionais quando associadas às informações emocionais tendem a melhorar o desempenho em tarefas explícitas (Kleinsmith \& Kaplan, 1963; Maratos \& Rugg, 2001). Ademais, na fase de codificação, fatores emocionais poderão potencializar estes processos de codificação melhorando, assim, o desempenho subseqüente; além disto, informações que concentram o foco da atenção podem aumentar o desempenho para estes traços emocionais em detrimento de aspectos periféricos (Christianson \& Loftus, 1991; Porter, Spencer, \& Birt, 2003). Porter et al. (2003), utilizando o paradigma da falsa informação, chegaram à conclusão de que estímulos com valência carregada negativamente são mais propensos à produção de falsas memórias do que estímulos neutros ou positivos. Os autores defendem que a memória humana é construtiva por natureza e que as falsas memórias são causadas por fatores externos que influenciam especialmente a decisão, ou seja, o monitoramento do indivíduo.

O estudo de Brainerd et al. (2008), que utilizou o paradigma DRM (Deese/Roediger/McDermott), do experimento pioneiro de Roediger e McDermott (1995), investigou a influência da valência de listas de palavras na produção de falsas memórias. Os resultados indicaram que a identificação de distratores críticos (palavras que representam a essência semântica das listas, mas que não foram apresentadas para memorização) ocorreu mais em listas de palavras com valência negativa, neutras e positivas, respectivamente. Os autores explicaram este resultado à luz da teoria do Traço Difuso (Brainerd \& Reyna, 2005), que descreve a memória humana incluindo dois sistemas independentes: o literal (armazena traços específicos do evento) e o de essência (armazena o significado geral do evento). Esta teoria sugere que a ocorrência de falsas memórias nas listas negativas é causada pela redução da habilidade dos participantes em usar traços específicos da palavra, que são mais susceptíveis à interferência. Ademais, tanto as listas negativas quanto neutras produziram falsas memórias a partir de ativações do conteúdo semântico. Por outro lado, as falsas recordações nas listas positivas, nas quais os índices de familiaridade foram quase zero, sugerem que os distratores críticos tiveram critérios diferentes de recuperação, ou seja, para itens com carga emocional positiva um sistema diferente de processamento estaria envolvido resultando em altos índices de recuperação "fantasma", que consiste na lembrança de um item que não foi apresentado, compreendendo fatores que vão além do processamento semântico, como os fatores emocionais.

Pesquisas mostraram que o tipo de processamento, consciente ou automático durante a codificação, afeta a produção de falsas lembranças (Cotel et al., 2008; Huang \& Janczura, 2008). Zeelenberg, Boot e Pecher (2005) demonstraram que a ativação do distrator crítico durante a fase de estudo não é necessária para se produzir falsos reconhecimentos. Resultados semelhantes foram relatados em Huang e Janczura (2008), no qual se comparou a ocorrência de falsas memórias em testes diretos e indiretos, evidenciando que mesmo em situações de priming houve uma influência das palavras relacionadas não estudadas no teste de identificação perceptual. Em outras palavras, não existe a necessidade do distrator crítico ser processado conscientemente para ativá-lo e produzir falsas memórias. Os resultados desses estudos mostraram que é possível observar falsas memórias quando o distrator crítico não é armazenado conscientemente durante a fase de estudo. Isto sugere um problema para as teorias que tentam explicar o fenômeno das falsas memórias apoiando-se na hipótese do espalhamento da ativação semântica (McDermott \& Watson, 2001). Esta hipótese argumenta que as falsas memórias ocorrem em função da ativação espalhada, entretanto, é plausível questionar se a ativação teria uma orientação unicamente semântica, ou poderia disparar em função de aspectos sintáticos ou, ainda, dependendo do estímulo, a ativação poderia ser dirigida emocionalmente (i.e., valência)? Além disto, até que ponto pode-se filtrar ou discriminar os componentes notadamente semânticos, pictóricos, emocionais ou sensoriais dos estímulos? Teoricamente é razoável supor que a ativação compreenda diferentes naturezas de sistemas mnemônicos interconectados. Evidências sugerem que o tipo de informação poderá determinar a rede associativa a ser ativada e codificada que, por sua vez, influenciará o processo de recuperação (Brainerd et al., 2008; Huang \& Janczura, 2008; Porter et al., 2003).

Seguindo esta linha de raciocínio, como se comportará o fenômeno das falsas memórias em um paradigma experimental que envolvesse o tipo de processamento (consciente ou não), a manipulação da emoção (valência) dos estímulos memorizados e, ainda, avaliasse estes fatores em diferentes contextos emocionais? Será que a apresentação de uma lista de palavras com valência negativa, estudada conscientemente em um contexto de valência negativa, maximizaria a produção de falsas memórias? O cruzamento experimental destes fatores permitirá avaliar a contribuição independente de cada variável assim como suas interações em diferentes contextos emocionais na produção de falsas memórias. Além disto, ajudará a esclarecer a relação entre a rapidez da codificação associada aos aspectos emocionais do estímulo no fenômeno das falsas memórias e, assim, 
contribuir para as teorias existentes. Se for verificado um índice maior de falsas memórias em listas com valência negativa, apresentadas rapidamente ao olho, em contextos de valências desagradáveis, então precisaríamos aprimorar nossas teorias e paradigmas atuais no sentido de contemplar variáveis emocionais nas metodologias experimentais. E, se for observado falso reconhecimento com distratores críticos carregados emocionalmente em tarefas que envolvam processamento automático, fortalecer-se-á o argumento de que este processo pode ser influenciado por conteúdo emocional associado à valência. Este dado pioneiro abrirá um campo novo de estudos sobre falsas memórias e tarefas implícitas que, até o momento, tem sido tangencialmente contemplado nos modelos de memória.

Este estudo investigou o efeito da valência emocional no paradigma DRM, manipulando as cargas afetivas das listas de palavras a serem memorizadas, assim como o contexto emocional inserindo, entre as listas do paradig$\mathrm{ma}$, fotografias carregadas emocionalmente que foram selecionadas do International Affective Picture System IAPS (Lang, Bradley \& Cuthbert, 1999; Lang, Greenwald, Bradley, \& Hamm, 1993; Lang \& Ohman, 1988; Ribeiro, Pompéia, \& Bueno, 2005). Esta manipulação no DRM é inédita e representa um avanço metodológico no estudo das falsas memórias.

O objetivo das manipulações experimentais propostas foi compreender a interação entre as valências emocionais oriundas de diferentes fontes (i.e., contexto e lista de palavras), assim como a contribuição individual destas fontes. Três variáveis foram manipuladas: a valência das listas de DRM (negativa, positiva ou neutra), o tipo de processamento (consciente ou automático) e a valência do contexto emocional (desagradável, agradável ou neutro). Quatro foram as hipóteses testadas: primeiro, índices superiores de falsas memórias ocorrerão em tarefas que demandam maior participação de processamento consciente, porque um número maior de ativações em redes diferenciadas (emocionais e semânticas) necessita de um maior tempo para que sejam processadas, mesmo de forma equivocada. Segundo, haverá um aumento no fenômeno das falsas memórias se o contexto tiver carga emocional; pesquisas mencionadas, anteriormente, apontam para este resultado, quando a valência é manipulada, ocorrerá uma produção considerável de falsas recordações (Porter et al., 2003; Wright, Startup, \& Mathews, 2005). A explicação desta expectativa é que a distorção de memória se deverá ao impacto emocional das figuras frente aos processos atencionais no momento da codificação e, também, na tomada de decisão do participante, ou seja, o indivíduo será induzido a processar, além da rede semântica, um contexto emocional com informações dramáticas. Terceiro, haverá maior falsificação de memória nas listas DRM com cargas negativas. Esta expectativa se apóia na hipótese de que estas listas ativarão uma rede de nódulos emocionais paralelos ao da rede semântica, aumentando a interferência e fortalecendo a distratora crítica. Além disso, itens negativos podem ser processados por vias distintas dos positivos e neutros, ou seja, um sistema apetitivo para as positivas e um aversivo para as negativas (Lang, 1995). Quarto, ocorrerá uma maior produção de falsas recordações na condição em que os participantes processarem conscientemente as listas negativas em um contexto desagradável, e também na condição de processamento automático. Isso se deverá à combinação dos efeitos do contexto desagradável e da carga negativa das listas quando o processamento for consciente. Isto sobrecarregará os sistemas envolvidos (interferência entre a rede emocional, semântica e perceptual) aumentando, assim, a ocorrência de falsas memórias. Outro fator seria a questão das vias distintas de valência explicadas na hipótese anterior. A manipulação do tipo de processamento (i.e., consciente ou automático) foi produzida estabelecendo diferentes intervalos de tempo para a apresentação dos estímulos. Apresentações extremamente breves (p.ex., 40 $\mathrm{ms}$ ) reduzem drasticamente a possibilidade do indivíduo reconhecer o estímulo apesar de ele ser capaz de detectar o seu aparecimento, ou seja, ele tem consciência de que algo foi projetado na tela do computador mas não consegue identificar o que viu. Já tempos mais longos (p.ex., 1500 $\mathrm{ms}$ ) permitem que o sujeito conscientemente identifique qual é a palavra apresentada.

\section{Método}

\section{Participantes}

Participaram voluntariamente do estudo 150 alunos universitários, selecionados por conveniência, sendo 108 mulheres e 42 homens na faixa etária entre 18 e 41 anos (Média $=24, D P=4,03$ ).

\section{Delineamento}

Foi aplicado um delineamento fatorial misto $(2 \times 3 \times 3)$ sendo os fatores tipo de processamento (consciente/1500 $\mathrm{ms}$ ou automático $/ 40 \mathrm{~ms}$ ) e valência do contexto emocional (desagradável, agradável ou neutro) manipulados entre-sujeitos, e carga afetiva das listas DRM (negativa, positiva ou neutra) manipulada intra-sujeitos. Seis grupos de participantes, cada um com 25 indivíduos, foram constituídos. Cada participante foi alocado, aleatoriamente, a uma condição experimental sendo que a ordem das condições era randômica. A variável dependente foi o desempenho dos participantes, no teste de reconhecimento, medido por meio da proporção de acertos (responder "Sim" para itens estudados) e de alarmes falsos (responder "Sim" para itens não estudados), no qual se avaliou a produção de falsas memórias.

\section{Materiais}

$\mathrm{Na}$ fase de estudo foi utilizado um total de 12 listas de palavras semanticamente relacionadas do DRM extraídas de Stein, Feix e Rohenkohl (2006). Cada nível do fator carga afetiva (negativa, positiva e neutra) teve 4 listas, sendo que a variável foi definida operacionalmente através 
Huang, T. P. \& Janczura, G. A. (2013). Contexto Emocional Negativo e Processamento Consciente na Produção de Falsas Memórias em Tarefas de Reconhecimento.

do valor da valência das listas, que varia de 1 a 9 . Os índices de valência foram os seguintes: listas positivas - Média $=8,38(D P=0,22)$; listas negativas - Média $=1,48(D P$ $=0,14)$, e listas neutras - Média $=5,12(D P=0,33)$. O alerta das listas foi controlado sendo as médias $4,86(D P$ $=0,80), 5,58(D P=0,28)$ e $5,03(D P=0,55)$ para as listas positivas, negativas e neutras, respectivamente. A dimensão alerta da emoção foi controlada uma vez que não era objetivo avaliar a sua influência na produção de falsas memórias. Uma ANOVA univariada foi calculada para verificar se havia diferença significativa entre o alerta das três categorias de listas utilizadas. A ANOVA revelou não haver diferença entre os alertas das listas, $F(2,15)=2,441$, $M S=0,847, p=0,121$. Cada lista continha 15 palavras associadas, totalizando 180 itens que foram apresentadas na fase de estudo.

Além disso, foram utilizadas doze fotografias do IAPS para cada uma dos três níveis de valência (desagradável, agradável e neutro) e mais uma foto para o treino. Os valores da valência das fotografias agradáveis, desagradáveis e neutras foram, respectivamente, $7,82,(D P=0,55), 1,8$ $(D P=0,46)$ e $4,8(D P=0,51)$. Os valores de alerta das fotografias agradáveis, desagradáveis e neutras foram 6,58 $(D P=0,27), 6,89(D P=0,27)$ e $6,71(D P=0,40)$, respectivamente. $\mathrm{O}$ alerta das fotografias também foi controlado pela mesma razão anteriormente apontada para o controle deste nas palavras. Nenhuma diferença estatisticamente significativa foi detectada entre os alertas das fotografias $[F(2,21)=1,804, M S=0,197, p=0,189]$.

No teste de reconhecimento foram utilizadas 72 palavras, divididas em três grupos: 36 palavras alvos, ou seja, palavras que foram estudadas; 12 palavras distratores críticos e 24 palavras novas, ou seja, não relacionadas semanticamente. Os três grupos tinham o mesmo número de palavras correspondentes aos três níveis da valência (positiva, negativa e neutra). Foram evitadas as listas do DRM cujo distrator crítico era caracterizado como por um rótulo emocional (por exemplo, alegria, medo), com a finalidade de se evitar um possível viés experimental.

\section{Procedimentos}

As sessões experimentais duraram, em média, 18 minutos e foram conduzidas em ambiente isolado e os participantes testados individualmente. Todos os cuidados éticos foram observados. As instruções explicaram aos participantes que se tratava de um estudo sobre memória e a tarefa era memorizar palavras e fotografias que seriam apresentadas na tela do computador. As listas de palavras e fotografias foram apresentadas pelo programa SuperLab Pro. Houve uma fase de treino, onde se apresentou aos participantes uma seqüência de 6 palavras. $\mathrm{O}$ tempo de apresentação foi de $1500 \mathrm{~ms}$ ou $40 \mathrm{~ms}$, com atualização na taxa de $60 \mathrm{~Hz}$, para cada estímulo dependendo do grupo experimental. Na metade da apresentação das palavras treino apareceu uma fotografia no centro da tela por 10 segundos que foi seguida por uma tela branca. Todas as palavras foram apresentadas na fonte System, tamanho
16. Na fase de estudo, o experimentador leu uma breve instrução específica que solicitou ao participante ler, observar e memorizar com muita atenção as palavras e fotografias. Após isso, iniciou-se a apresentação das 12 listas de palavras em uma ordem randômica. Um intervalo de 10 segundos separou a apresentação de cada lista. Durante este intervalo, uma fotografia apareceu no centro do monitor; logo depois, foi apresentada a próxima lista $\mathrm{e}$, assim, sucessivamente, intercalando listas e fotografias até a apresentação completar as doze listas e dez fotografias. A apresentação das fotografias também ocorreu em uma ordem randômica. As quinze palavras de cada lista foram apresentadas uma de cada vez, numa ordem fixa e decrescente de associação semântica com o distrator crítico, ou seja, as primeiras palavras de cada lista foram mais fortemente associadas semanticamente com o distrator crítico não apresentado do que as últimas palavras da lista, de acordo com critérios clássicos do paradigma DRM (Roediger \& McDermott, 1995). O tempo do intervalo entre estímulos foi de $32 \mathrm{~ms}$ em todas as condições experimentais. A escolha deste intervalo foi baseada em estudos experimentais com o objetivo de maximizar as taxas de falsas memórias (Huang \& Janczura, 2008; McDermott \& Watson, 2001). Após a apresentação da última lista, a tela do computador voltou a permanecer em branco e os participantes foram instruídos a realizar uma tarefa diferente, dando início à atividade interpolada, que consistiu em executar, durante dois minutos, operações aritméticas de multiplicação, adição e subtração. A função da atividade interpolada foi neutralizar os efeitos de recência e primazia da ordem de apresentação dos estímulos do estudo. A tela do computador permaneceu em branco durante esta fase. Em seguida, os participantes foram submetidos a um teste de reconhecimento de 72 itens. As palavras foram apresentadas em uma ordem randômica na tela, uma de cada vez, e os participantes liam em voz alta a palavra e respondiam teclando uma entre duas teclas possíveis: SIM ou NÃO. Os participantes deveriam responder SIM se julgassem ter visto a palavra ou responder NÃO se julgassem não tê-la lido. O experimentador se posicionou atrás do participante durante esta fase de teste. O tempo de execução do teste era livre.

\section{Resultados}

A avaliação do desempenho dos participantes no paradigma DRM considera três categorias de palavras: palavras apresentadas na fase de estudo (PE), distratores críticos não apresentados (PC) e palavras novas não apresentadas (PN) que não têm relação semântica com as palavras estudadas e nem os distratores críticos. Os dados brutos dos acertos foram transformados em porcentagem, uma vez que os números de palavras em cada grupo são diferentes. As falsas memórias são observadas quando os participantes produzem consideravelmente mais respostas "SIM" para PC do que PN e quando a quantidade de respostas "SIM" entre PC e PE é semelhante. 
O tempo de apresentação das palavras produziu um efeito significativo $[F(1,144)=809,78, M S=374160,47$, $p<0,001]$. No tempo de $1500 \mathrm{~ms}$, as médias de respostas SIM foram $68 \%$ para PC, $72 \%$ para PE e $30 \%$ para PN. No tempo de $40 \mathrm{~ms}$ as médias foram $25 \%$ para PC, $28 \%$ para PE e $18 \%$ para PN. Um teste $t$ com correção para Bonferroni indicou que a diferença entre as médias PC e $\mathrm{PE}$, independentemente do tempo de apresentação, não foi significativa e entre PC e PN foi significativa $(p<0,001)$ caracterizando, assim, a ocorrência de falso reconhecimento. Ademais, registrou-se que a média de PC (68\%) no tempo lento foi significativamente superior ao tempo rápido $(25 \%)$, indicando um maior percentual de falsos reconhecimentos nesta condição. A valência das fotografias também influenciou significativamente o desempenho dos participantes, $[F(2,144)=7,779, M S=3594,39, p$ $<0,001]$. No contexto desagradável, as médias de resposta SIM foram 58\%, 55\%, 27\%, respectivamente, para PC, PE e PN. No contexto agradável, as médias foram $46 \%, 50 \%$, $26 \%$ para PC, PE e PN, respectivamente. E, no contexto neutro foram $46 \%, 48 \%, 23 \%$, respectivamente para $\mathrm{PC}$, $\mathrm{PE}$ e PN. Quando comparadas as médias de PC entre as três valências, através do teste $t$ com correção para Bonferroni, foram verificadas diferenças significativas entre PC do nível desagradável com os outros dois contextos. Não houve diferença significativa entre PC e PE em cada um dos três contextos. Estes resultados confirmam duas expectativas experimentais: primeiro, índices superiores de falsas memórias ocorreram em tarefas que demandassem maior participação de processamentos conscientes e, segundo, que haverá um aumento no fenômeno das falsas memórias se o contexto fosse emocionalmente carregado.

A ANOVA mista $(2 \times 3 \times 3 \times 3)$ entre tempo de apresentação (1500 ms e $40 \mathrm{~ms}$ ), valência das fotografias (agradável, desagradável e neutra), carga das listas (positiva, negativa e neutra) e tipo de palavras (PC, PE e PN) indicou um resultado significativo para o efeito principal da carga das listas, $[F(2,288)=12,19, M S=2861,13, p<0,001]$. Nas listas positivas as médias de SIM para PC, PE e PN foram, respectivamente, $45 \%, 49 \%$ e $23 \%$ e para as listas negativas foram $52 \%, 53 \%$ e $26 \%$. E, finalmente, para as listas neutras foram $44 \%, 50 \%$ e $25 \%$, respectivamente. Aplicando-se testes $t$ com correção para Bonferroni, verificou-se não haver diferenças significativas entre as médias de PC e PE das listas. Resultado oposto foi observado entre as médias de PC e PN $(p<0,01)$, indicando a ocorrência de falsas recordações. Quando comparadas as médias de PC para cada tipo de lista, verificou-se uma diferença significativa entre PC da lista negativa versus positiva e negativa versus neutra $(p<0,001)$, evidenciando uma maior produção de falsas memórias nas listas negativas. Uma ANOVA entre os fatores carga das listas e valência das fotografias, na condição de $1500 \mathrm{~ms}$, detectou uma interação significativa, $[F(2,74)=3,362, M S=658,33, p=0,04]$. O mesmo ocorreu com uma ANOVA na condição de $40 \mathrm{~ms},[F(2$, $74)=4,895, M S=1200,00, p=0,01]$. Observando as Figuras 1 e 2, nota-se que as maiores produções de PC e PE ocorreram no contexto desagradável e nas listas negativas. Esse efeito é representado por um "V" invertido nos dois gráficos, demonstrando uma maior produção de memória falsa nessas duas condições.

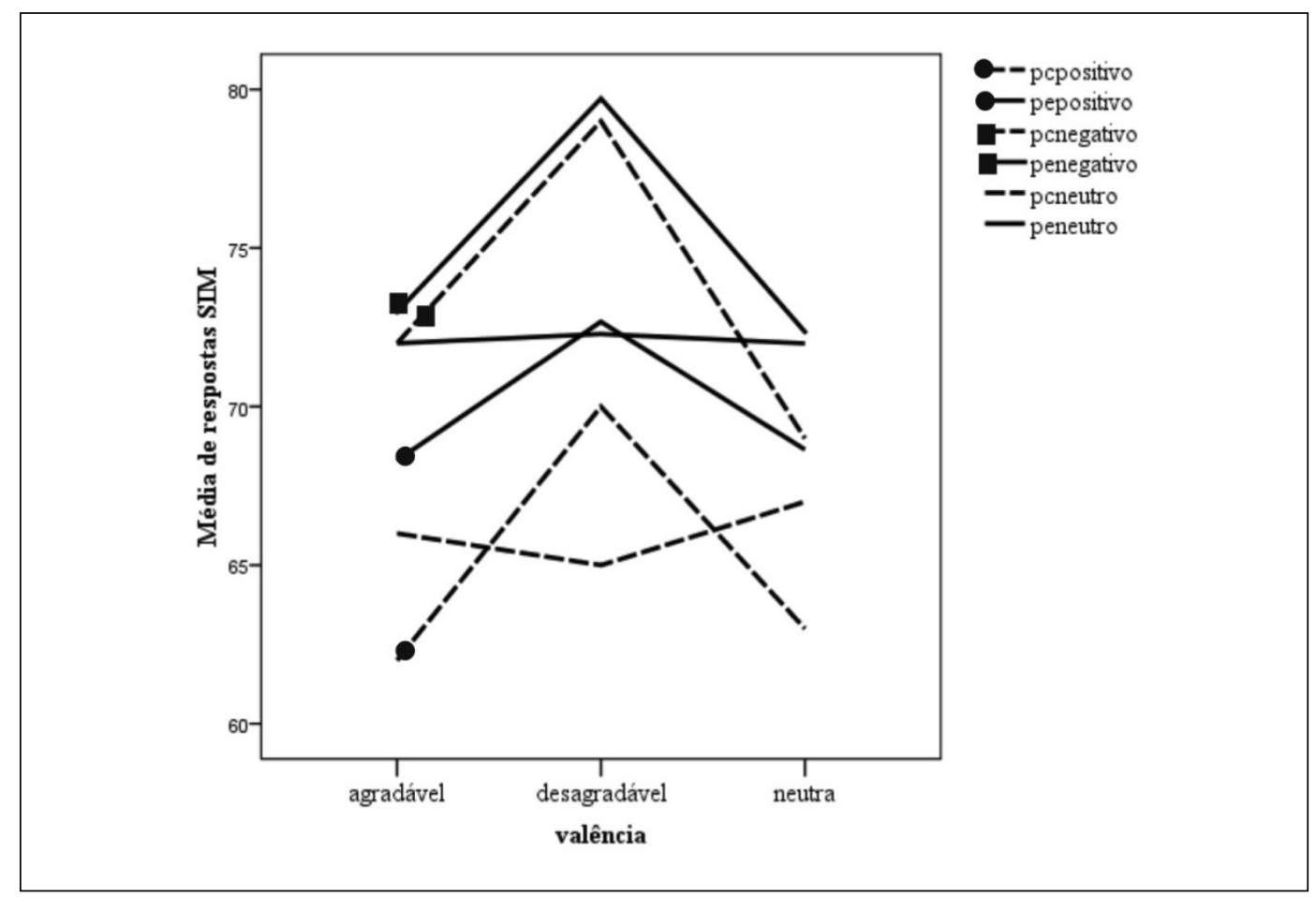

Figura 1. Média de acertos na condição de 1500 ms em função do tipo de palavra, carga das listas e valência. 
Huang, T. P. \& Janczura, G. A. (2013). Contexto Emocional Negativo e Processamento Consciente na Produção de Falsas Memórias em Tarefas de Reconhecimento.

As médias de PC nas listas negativas, no tempo de $1500 \mathrm{~ms}$, foram $80 \%, 72 \%$ e $69 \%$, respectivamente, para o contexto desagradável, agradável e neutro. O teste $t$ com correção para Bonferroni entre essas médias apontou uma diferença significativa apenas entre as médias de PC do contexto desagradável com o neutro $(p=0,04)$.

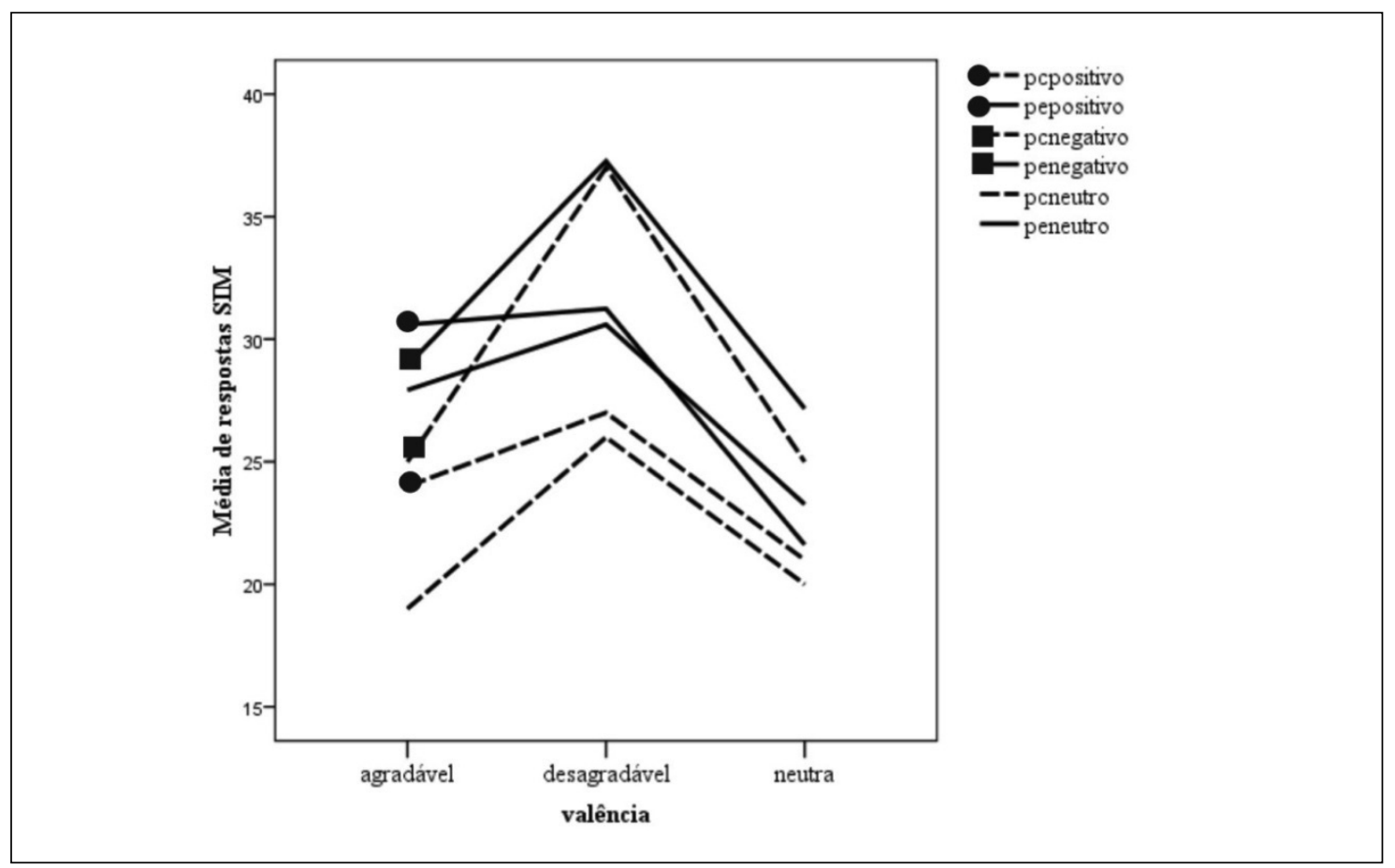

Figura 2. Média de acertos na condição de $40 \mathrm{~ms}$ em função do tipo de palavra, carga das listas e valência.

Efeito similar ocorreu no tempo de $40 \mathrm{~ms}$ para as médias de PC da lista negativa, $37 \%, 25 \%$ e $25 \%$ para contexto desagradável, agradável e neutro, respectivamente, no qual as diferenças significativas encontradas foram entre PC da condição desagradável em relação aos demais contextos. Estes resultados confirmam a quarta hipótese do estudo de que ocorreria maior índice de falsas memórias na condição de processamento consciente, valência negativa das listas e valência desagradável do contexto. Esse mesmo padrão aparece no tempo de 40 $\mathrm{ms}$, mas em uma magnitude mais discreta. Além disso, quando aplicado um teste $t$ são significativas as diferenças entre PC e PE das listas positivas, no tempo $1500 \mathrm{~ms}$ entre os contextos agradável ( $62 \%$ e $68 \%$ ) e neutro $(63 \%$ e $69 \%), p=0,04$. O mesmo padrão acontece no tempo $40 \mathrm{~ms}$ apenas no contexto agradável $(24 \%$ e $31 \%)$. Este padrão é mais evidente quando a carga das listas é neutra, no tempo $1500 \mathrm{~ms}$, pois ocorrem diferenças significativas nos contextos agradável $(69 \%$ e $62 \%)$ e neutro $(69 \%$ e $63 \%$ ). Isso indica uma produção menos evidente de falsas recordações nas listas positivas e neutras devido à influência do tipo de carga emocional das listas sugerindo que, quanto maior for a diferença entre as médias de PC e PE, menor a probabilidade de falsos reconhecimentos. Por outro lado, quanto menor for a diferença entre PC e $\mathrm{PE}$, maior é a probabilidade dos participantes tratarem PC como PE, ou seja, mais falsas memórias.
Finalmente, foi calculado o $d$ prime $\left(d^{\prime}\right)$ das respostas Valores maiores de d' indicam maior capacidade de discriminação entre tipos de estímulo, como alvos, distratores críticos e distratores não relacionados (Moscovitch \& Bentin, 1993; Snodgrass \& Corwin, 1988). No caso das falsas memórias, pode-se adaptar esta medida para dois tipos de $d^{\prime}: d^{\prime}$ 'verdadeiro, que consiste na equação $d^{\prime}(\mathrm{v})$ $=z(\mathrm{PE})-z(\mathrm{PN}) / 10$, e o $d^{\prime}$ falso cuja equação é $d^{\prime}(f)=$ $z(\mathrm{PC})-z(\mathrm{PN}) / 10$. Quanto maior o $d$ ' maior será o grau de sensibilidade de reconhecimento (seja falso ou verdadeiro). Na ANOVA mista aplicada ao $d$ ' falso, o fator carga das listas foi fonte de variação significativa, $[F(2,288)=$ $3,714, M S=1315,10, p=0,02]$. As médias dos índices de $d$ ' falso para as listas positivas foi 2,1 , para as negativas foi 2,5 e para as neutras foi igual a 1,9. Testes $t$ com correção para Bonferroni indicaram que houve diferença significativa entre os $d$ 's falsos da lista negativa e a neutra. O fator tempo de apresentação também produziu um efeito significativo, $[F(1,144)=176,41, M S=102278,59, p=$ $0,001]$. O índice para $1500 \mathrm{~ms}$ foi de 3,6 e para $40 \mathrm{~ms}$ foi igual a 0,6 . Esses resultados indicam que os participantes tiveram maior sensibilidade de discriminação nas listas negativas comparadas com as positivas e neutras. $\mathrm{Na}$ ANOVA aplicada ao $d$ ' verdadeiro, o único fator entre sujeitos que produziu um efeito significativo foi o tempo de apresentação, $[F(1,144)=570,07, M S=300819,00, p=$ 0,001 ], sendo as médias 4,1 para $1500 \mathrm{~ms} \mathrm{e} 0,1$ para $40 \mathrm{~ms}$. 


\section{Discussão}

Os achados corroboram a expectativa de que haverá uma maior produção de falsas memórias em função do aumento do intervalo de tempo (1500 ms), na qual exige-se o processamento consciente da informação na fase de codificação. Argumenta-se que maiores intervalos de tempo de apresentação permitem mais ativações de nódulos de informação e, conseqüentemente, um maior monitoramento. Se a situação exige um grande volume de processamentos em um curto espaço de tempo, haverá uma maior probabilidade de ocorrer falsas recordações. Quanto maior o tempo permitido para estudar uma palavra, maior será a participação de processos de monitoramento na fase de codificação (McDermott \& Watson, 2001). Os resultados são consistentes com achados recentes da literatura (Huang \& Janczura, 2008; McDermott \& Watson, 2001; Seamon, Luo, \& Gallo, 1998). Sabe-se que existe um intervalo ideal de apresentação das palavras para a ocorrência de falsas memórias que ocorre, aproximadamente, entre 1000 ms e 1500 ms (Huang \& Janczura, 2008; McDermott \& Watson, 2001). Acima disso, os falsos reconhecimentos começam a diminuir devido às estratégias mais eficientes de monitoramento; abaixo disso, não há tempo suficiente para se elaborar estratégias eficientes o suficiente a fim de se prevenir contra as falsas lembranças.

A segunda hipótese do estudo, de que haverá maior produção de falsas memórias se o contexto fosse emocionalmente carregado, foi confirmada pelos resultados. Primeiramente, a comparação entre as médias das PCs dos três níveis de valência produziu diferenças significativas na valência desagradável. Isso faz sentido, pois não podemos generalizar o raciocínio de que ocorrerão falsas memórias independentemente do tipo de contexto emocional. A questão é em qual tipo de contexto emocional será observada uma taxa superior de falsos reconhecimentos. Os resultados apontam que é no contexto desagradável. Por exemplo, quando consideramos o contexto desagradável, tempo lento e lista negativa, verificamos uma ocorrência maior de falsos reconhecimentos. Sugere-se que nos contextos de valência desagradável a rede de informações tenha produzido maiores ativações e, conseqüentemente, maiores níveis de monitoramento da informação na fase de codificação diminuindo, discretamente, os julgamentos que pudessem dirimir os erros.

Constatou-se, também, que houve maior produção de falsas memórias em listas DRM com valência negativa do que positiva ou neutra. Encontrou-se uma diferença significativa na produção de falsos reconhecimentos das listas negativas em comparação com as positivas e neutras, mas não entre as positivas e neutras. A Figura 1 ilustra que não houve diferença significativa entre PC e PE nas listas negativas, ou seja, uma indicação clássica da ocorrência de falsos reconhecimentos. Esta diferença é mais evidente do que as médias para essas palavras nas listas positivas e neutras. $\mathrm{O}$ resultado sustenta que a carga afetiva negativa das listas aumenta a produção de falsas memórias. Uma explicação plausível para esses resultados é que a cognição humana tende a elaborar mais informações de caráter negativo, em processamentos que exigem informações semânticas e autobiográficas. Essa elaboração significa mais ativações nas redes semânticas e autobiográficas, induzindo a um erro de decisão pela sobrecarga de informações processadas em um tempo limitado (Kensinger \& Corkin, 2004). É plausível que itens com carga negativa ativem uma maior quantidade de nódulos de informações (em comparação aos itens positivos e neutros) e, com isso, sobrecarregem o sistema de codificação (que teria um limite próximo de $1500 \mathrm{~ms}$ ) e, posteriormente, de monitoramento comprometendo a identificação correta e acurada da informação estudada. Ademais, esses resultados podem ser explicados pela idéia de dissociação entre memórias verdadeiras e falsas. Segundo a teoria do Traço Difuso (Brainerd \& Reyna, 2005), falsas recordações são derivadas da recuperação de traços de essência, enquanto as memórias verdadeiras são oriundas majoritariamente de traços literais. Estendendo a interpretação dessa hipótese teórica, pode-se afirmar de acordo com os resultados encontrados que uma lógica dissociativa também faz sentido em relação à valência das informações verbais. Em outras palavras, itens negativos podem ser processados por vias distintas dos positivos e neutros. Esta idéia é sustentada pela teoria de Lang (1995), que prevê a existência de sistemas distintos de processamento para informações negativas e positivas, ou seja, um sistema apetitivo para as positivas e um aversivo para as negativas. Os achados corroboram esta ideia, pois se encontrou uma diferença significativa entre as cargas positiva e negativa. Os resultados obtidos podem, também, ser explicados pela hipótese da teoria de Rede (McDermott \& Watson, 2001). É razoável supor que a informação humana tratada como uma rede semântica necessite interagir com outras redes, nesse caso, as redes emocionais. Pode-se inferir a presença de uma rede emocional capturada, por exemplo, pelo fator valência das informações que estão sendo ativadas e que esta interação (semântica, valência, alerta, entre outras) durante a codificação influencia as respostas do indivíduo. Especula-se que estas ativações não sejam aleatórias e que existe alguma prioridade para a sua ocorrência. Os resultados desse estudo demonstram que, considerando o componente emocional, a prioridade é influenciada pela valência desagradável e pela carga negativa das listas de palavras.

Finalmente, os resultados revelaram uma diferença significativa nas sensibilidades do $d$ ' falso das listas e tempo e do $d$ ' verdadeiro do tempo. No caso do $d$ ' falso, que indica o grau de sensibilidade de decisões falsas, isso sugere uma dissociação no processamento de discriminação entre itens neutros e negativos. Os achados mostraram que o $d$ ' falso das listas negativas apresentou um índice maior do que das listas neutras. Isto sugere que os participantes foram mais sensíveis na discriminação do $d$ ' falso das listas negativas comparadas com as neutras. Este dado confirma as afirmações mencionadas anteriormente de 
Huang, T. P. \& Janczura, G. A. (2013). Contexto Emocional Negativo e Processamento Consciente na Produção de Falsas Memórias em Tarefas de Reconhecimento.

que informações negativas exigem maior processamento de elementos emocionais e redes cognitivas. Além disso, os resultados de $d$ prime corroboram, parcialmente, estudos recentemente realizados sobre fatores emocionais das listas DRM utilizando a abordagem da detecção de sinal (Brainerd et al., 2008; Santos, 2006), que relataram índices maiores de $d$ ' falso para listas negativas. Contudo, o $d$ ' verdadeiro não foi afetado pelas cargas das listas, demonstrando que o tipo de informação é crítico para a compreensão da noção de dissociação entre sistemas de memórias, ou seja, é plausível a ocorrência de um sistema duplo e independente na produção de falsas memórias, mas esses dados podem, também, indicar uma interação ou conexão entre sistemas diferentes.

\section{Considerações Finais}

O presente estudo teve o objetivo de entender a interação entre o efeito da valência do contexto, das cargas das listas de DRM com o processamento consciente e automático. Foi observada uma interação entre esses fatores e, além disso, verificou-se que a valência do contexto e das listas exerce uma influência significativa na produção de falsos reconhecimentos no paradigma DRM modificado.

Pesquisas na área de emoção têm demonstrado que contextos emocionais considerados dramáticos têm um efeito primário de maior impacto sobre contextos emocionais não tão dramáticos (Porter et al., 2003; Porter, Yuille, \& Lehman, 1999). Os contextos emocionais considerados "neutros" não produziram tantas falsas memórias quanto os emocionais, por não ativarem tantas redes associadas de natureza semântica ou emocional. Resultados consistentes com esta hipótese podem ser verificados na literatura (Kensinger \& Corkin, 2003; Lang, 1995; Porter et al., 2003). O presente estudo confirmou este achado e estendeu esta noção combinando fatores emocionais em uma forma mais complexa e diferenciada: manipulando-se os fatores valência de diferentes tipos de informações e associando-os em uma mesma fase de estudo. Pode-se sugerir, apoiando-se em nossos achados, que existem indicações de sistemas e processamentos diferenciados para componentes emocionais distintos. Há teorias que defendem a codificação de informação através de vários sistemas diferentes de processamento (Brainerd \& Reyna, 2005; Lang, 1995). Há a necessidade de entender melhor o funcionamento dessas diferentes vias de processamentos para diferentes fatores (valência, rapidez de codificação). Contudo, é de extrema relevância a compreensão sobre como os diferentes sistemas (semântico e emocional/valência) se interligam no complexo funcionamento da memória humana. Sistemas que funcionam de uma forma independente, mas que podem se conectar dependendo do tipo de informação e da natureza da valência do contexto.

A partir dos resultados do presente estudo, é razoável especular que o efeito da valência provoca um forta- lecimento de certas conexões semânticas que podem, dependendo do contexto, favorecer a produção de falsas memórias. Por exemplo, uma pessoa que precisa memorizar uma informação negativa, em um contexto de valência desagradável, poderá sofrer consideravelmente mais falsas interpretações comparadas a um contexto neutro com informações, relativamente, "usuais". É plausível supor que contextos e informações desagradáveis aumentem a quantidade de ativações nas diversas redes e esquemas cognitivos que podem sobrecarregar o sistema de codificação e conseqüentemente influenciar a memória.

Uma contribuição inédita deste estudo foi investigar a interação da valência oriunda de duas fontes, durante a fase de estudo, associadas ao DRM e IAPS. Nota-se que a combinação entre as valências desagradável e negativa apresentou um efeito aditivo que aumentou a ocorrência de falsos reconhecimentos da palavra distratora crítica. $\mathrm{O}$ aumento das ativações com conotação negativa e informações com naturezas diferentes podem ocorrer conjuntamente produzindo maiores processamentos de informações e de componentes emocionais. Estes interferem no armazenamento das informações estudadas a ponto de se produzir falsificações consideráveis. Infere-se que o aspecto negativo produz maiores ativações interferentes no sistema de memória comparadas aos aspectos positivo e neutro. Outra contribuição deste estudo foi demonstrar que o processamento consciente representa uma peça fundamental no efeito de valência descrito acima, mas este efeito tem um padrão similar, numa magnitude menor, em condições de processamento automático. Apesar de se registrar uma diminuição na produção de falsas memórias, observou-se um padrão semelhante nos tempos rápidos, porém, em menor magnitude. Isso pode ser verificado nos resultados, o padrão de " $\mathrm{V}$ " invertido dos resultados se repetiu no tempo de $40 \mathrm{~ms}$, apenas em uma magnitude menor. Isto, também, pode indicar uma espécie de processador cognitivo responsável por identificar aspectos de valência da informação já em um nível "não consciente", e não apenas no âmbito do processamento consciente, como algumas pesquisas afirmam (Kensinger \& Schacter, 2006). Em outras palavras, o sistema responsável pela coesão ou falsificação mnemônica não necessariamente funciona em compartimentos, claramente definidos. A noção de rede emocional e o fortalecimento de suas conexões pode ser um modelo de explicação bastante razoável para esclarecer a relação funcional entre as falsas memórias e a valência.

Por fim, futuras pesquisas poderão concentrar seus estudos focalizando duas questões: a primeira se relaciona a interação contextual entre a valência e o alerta, por exemplo, em uma situação dramática, utilizando informações de mesma natureza. As decisões das pessoas tendem a ser influenciadas mais pela valência ou alerta? A segunda questão é metodológica: é necessário produzir novas listas DRM que torne possível a manipulação simultânea dos fatores alerta e valência; isto permitiria investigar a interação entre valência e alerta em um mesmo contexto emocional. 


\section{Referências}

Brainerd, C. J., \& Reyna, V. F. (2005). The science of false memory. Oxford, UK: Oxford University Press.

Brainerd, C. J., Stein, L. M., Oliveira, R. A., Rohenkohl, G., \& Reyna, V. F. (2008). How does negative emotions cause false memories? Psychological Science, 19, 919-925.

Christianson, S. A., \& Loftus, E. F. (1991). Memory for traumatic events. Applied Cognitive Psychology, 1, 225-239.

Cotel, S. C., Gallo, D. A., \& Seamon, J. G. (2008). Evidence that nonconscious processes are sufficient to produce false memories. Consciousness and Cognition, 17, 210-218.

Doerksen, S., \& Shimamura, A. P. (2001). Source memory enhancement for emotional words. Emotion, 1, 5-11.

Ellis, H. C., \& Moore, B. A. (1999). Mood and memory. In T. Dalgleish \& M. J. Power (Eds.), Handbook of cognition and emotion (pp. 191-210). New York: John Wiley and Sons.

Gallo, D. A. (2006). Associative illusions of memory. New York: Psychology Press.

Kensinger, E. A., \& Corkin, S. (2003). Memory enhancement for emotional words: Are emotional words more vividly remembered than neutral words? Memory and Cognition, 31, 1169-1180.

Kensinger, E. A., \& Corkin, S. (2004). The effects of emotional content and aging on false memories. Cognitive, Affective and Behavioral Neuroscience, 4, 1-9.

Kensinger, E. A., \& Schacter, D. L. (2006). Processing emotional pictures and words: Effects of valence and arousal. Cognitive, Affective, \& Behavioral Neuroscience, 6, 110-126.

Kleinsmith, L. J., \& Kaplan, S. (1963). Paired-associate learning as a function of arousal and interpolated interval. Journal of Experimental Psychology, 65, 190-203.

Huang, T. P., \& Janczura, G. A. (2008). Processos conscientes e inconscientes na produção de falsas memórias. Psicologia: Teoria e Pesquisa, 24, 347-354.

Lang, P. J. (1995). The emotion probe: Studies of motivation and attention. American Psychologist, 50, 372-385.

Lang, P., Bradley, M., \& Cuthbert, B. N. (1999). International Affective Picture System (IAPS): Instruction Manual and Affective Ratings. Gainesville, FL: National Institute of Mental Health Center for the Society of Emotion and Attention, University of Florida.

Lang, P., Greenwald, M. K., Bradley, M., \& Hamm, A. O. (1993). Looking at pictures: Affective, facial, visceral and behavioral reactions. Psychophysiology, 30, 261-273.

Lang, P., \& Ohman, A. (1988). The International Affective Picture System (IAPS) [Photographic slides]. Gainesville, FL: The Center for Research in Psychophysiology, University of Florida.

Maratos, E. J., Allan, K., \& Rugg, M. D. (2000). Recognition memory for emotionally negative and neutral words: An ERP study. Neuropsychologia, 38, 1452-1465.

Maratos, E. J., \& Rugg, M. D. (2001). Eletrophysiological correlates of the retrieval of emotional and non-emotional context. Journal of Cognitive Neuroscience, 13, 877-891.

McDermott, K. B., \& Watson, J. M. (2001). The rise and fall of false recall: The impact of presentation duration. Journal of Memory and Language, 45, 160-176.

Moscovitch, M., \& Bentin, S. (1993). The fate of repetition effects when repetition approaches chance. Journal of Experimental Psychology: Learning, Memory \& Cognition, 19, 148-158.
Porter, S., Spencer, L., \& Birt, A. R. (2003). Blinded by emotion? Effect of the emotionality of a scene on susceptibility to false memories. Canadian Journal of Behavioral Science, 35, 165-175.

Porter, S., Yuille, J. C., \& Lehman, D. R. (1999). The nature of real, implanted and fabricated memories for emotional childhood events: Implications for the recovered memory debate. Law and Human Behavior, 23, 517-537.

Ribeiro, R. L., Pompéia, S., \& Bueno, F. A. B. (2005). Comparação entre normas brasileiras e norte-americanas do International Affective Picture System (IAPS). Revista Brasileira de Psiquiatria, 27, 208-215.

Roediger, H. L., \& McDermott, K. B. (1995). Creating false memories: Remembering words not presented in lists. Journal of Experimental Psychology: Learning, Memory and Cognition, 21, 803-814.

Santos, R. F. (2006). Abordagem experimental no estudo das emoções e falsas memórias (Dissertação de mestrado, Pontifícia Universidade Católica do Rio Grande do Sul, Porto Alegre, RS, Brasil).

Seamon, J. G., Luo, C. R., \& Gallo, D. A. (1998). Creating false memories of words with or without recognition of list items. Psychological Science, 9, 20-26.

Snodgrass, J. G., \& Corwin, J. (1988). Pragmatics of measuring recognition memory: Applications to dementia and amnesia. Journal of Experimental Psychology: General, 117, 34-50.

Stein, L. M., Feix, L. F., \& Rohenkohl, G. (2006). Avanços metodológicos no estudo das falsas memórias: Construção e normalização do procedimento de palavras associadas à realidade brasileira. Psicologia: Reflexão e Crítica, 19, 166-176.

Zeelenberg, R., Boot, I., \& Pecher, D. (2005). Activating the critical lure during study is unnecessary for false recognition. Consciousness and Cognition, 14, 316-326.

Wright, D. B., Startup, H. M., \& Mathews, S. A. (2005). Mood, dissociation and false memories using the DRM procedure. British Journal of Psychology, 96, 283-293.
Recebido: 06/06/2011 $1^{a}$ revisão: $02 / 04 / 2012$ $2^{a}$ revisão: $15 / 05 / 2012$ Aceite final: 07/08/2012 